\title{
Prevalence of Staphylococcus aureus in Intensive care Units and Post Operative Ward as a possible source of Nosocomial infection: An experience of tertiary care hospital
}

\author{
Bhattarai P, Dhungel BA, Shah P, Amatya J \\ Think Tank Foundation, Jorpati, Nepal \\ Kathmandu College of Science and Technology, Kalimati, Kathmandu, Nepal
}

\begin{abstract}
Background and Objectives: Hospital Acquired Infection (HAI) also called nosocomial infection is a global problem. It also acts as a leading cause of death and increased morbidity among hospitalized patients. Staphylococcus aureus is one of the commonest pathogens causing HAI and is also closely associated with human body. WHO study has shown highest prevalence of HAI in Intensive care unit (ICU), Neonatal care unit (NICU), Post operative ward (POW). Hence it becomes very imperative to check the prevalence of HAI. Therefore this study was carried out to determine the prevalence of $S$. aureus in hospital environment as a possible source for HAI.

Material and Methods: Environmental samples and blood samples of the patients were collected from ICU, NICU, POW using sterile cotton swabs during study period. Standard protocol was followed to isolate and identify $S$. aurues which was followed by antibiotic sensitivity tests.

Results: A total of 216 environmental samples were collected out of which $18.98 \%$ (41) samples showed the presence of $S$. aureus. It was also isolated from the blood samples of patients admitted to ICU $(7.5 \%)$ and NICU (6.8\%). None of the isolates from environmental samples were MRSA(methycillin resistant Staphylococcus aures).

Conclusion: S. aureus was found as a prominent environmental flora of hospital setting. Presence of these organisms in sensitive units like ICU, NICU, POW and the isolation of $S$. aureus from clinical samples indicates a possibility of HAI. It indicates the necessity of continuous surveillance of hospital environmental for quality control.
\end{abstract}

Key words: S. aureus, Nosocomial infection, HAI, ICU, NICU

INTRODUCTION

Hospital Acquired Infection (HAI), also called nosocomial infection is defined as an infection acquired in hospital by a patient who was admitted for a reason other than that infection [1]. This also includes infection acquired in the 
hospital but appearing after discharge and occupational infections among staffs working in the hospital [2]. Hospital acquired infection is a global problem. At any time over 1.4 million people worldwide suffer from infectious complications acquired in hospital [3]. A WHO report has shown that frequency of nosocomial infection in South East Asia is $10 \%$. In a study done in Nepal, the overall point prevalence of HAI is reported to be $2.4 \%$ [4].

Many factors like old age, underlying disease, chemotherapy, low level immunity, variety of invasive medical procedures promote nosocomial infection among hospitalized patients. This increases the complications of the treatment and may also act as a leading cause of death and increased morbidity among hospitalized patients [3]. Nosocomial infections can also spread among hospital staffs, visitors and may affect community. Emergence of drug resistant bacteria which can cause hospital acquired infection is an alarming state as the treatment and control of such multidrug resistant organism is extremely difficult [2].

Different microorganisms can cause hospital acquired infections. S. aureus is one of the commonest pathogens associated with HAI. The emergence of multi drug resistant $S$. aureus has become a major concern because of higher mortality due to hospital acquired MRSA (methycillin resistant Staphylococcus aures) infection [5].

S. aureus is commonly found in the environment including dust, water, air, feaces, clothing and utensils. It is also found closely associated with the human body. Nasal carriage of $S$. aureus has been identified as a risk factor for nosocomial infections [6]. Studies have shown that screening and eradiction of nasal carrier for $S$. aureus decreases the incidence of HAI [7].

HAI in sensitive units like Intensive care unit, Neonatal care unit, Post operative ward is even more frightening. WHO study has shown highest prevalence of HAI in such units. This may indicate further complication among critical patients in terms of cost, morbidity and mortality. Hence it becomes very imperative to check the prevalence of HAI.

Hence this study was performed as a surveillance of a tertiary care hospital environment in most vulnerable wards i.e. NICU, ICU, Post Operative Ward for one of the commonest organisms causing HAI i.e $S$. aureus.

\section{MATERIALS AND METHODS}

\section{Sample collection and processing:}

This study was carried out prospectively at tertiary care hospital (KIST medical college teaching hospital) during July to September 2010. Surface samples from inanimate objects (bed linings, floor and working table) were collected from ICU, NICU and Post Operative ward. The samples were collected using sterile dry cotton swab soaked in normal saline. Sampled swab sticks were transferred to Nutrient broth and incubated at 37 degree centigrade for 4 hours. Then culture was done on Nutrient agar and incubated at 37 degree centigrade for overnight. This was followed by staining, subculture and processing for isolation and identification of S.aureus. Antibiotic sensitivity test for the isolated $S$. aureus was done using Kirby Bauer disc diffusion method. Standard protocol and precaution were followed in the laboratory procedures.

\section{Quality control:}

Temperature chart was maintained for incubat or and hot air oven to monitor their performance. 
Quality check of culture media was done by incubating un-inoculated culture plate from each batch. All the reagents and antibiotic disc were checked for expiry date. Quality check was done using positive and negative control for the biochemical reagents used for the tests. Control strain of S. aureus (ATCC 25923) was used along with the suspected Staphylococcus spp strain to increase the validity of isolation and identification.

\section{RESULTS}

A total of 216 environmental samples were collected during the study period from ICU, NICU, Post operative ward, out of which $18.98 \%$ (41) samples showed the presence of $S$. aureus.

Fig 1: Percentage wise distribution of $S$. aureus among different wards

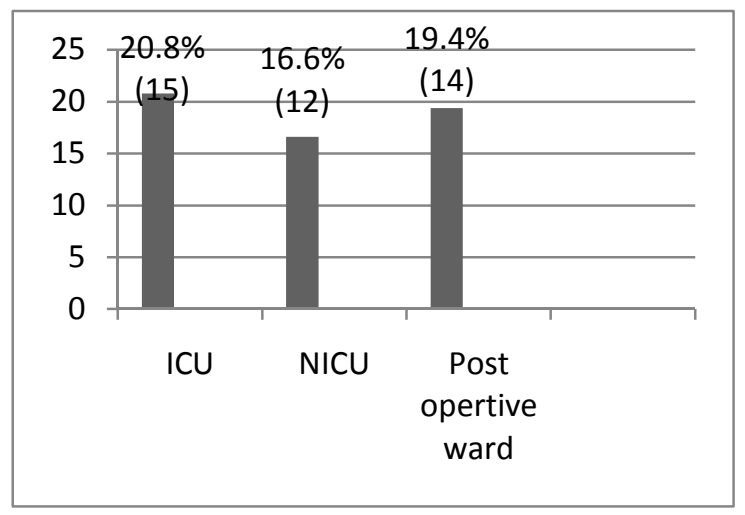

Total of 24 samples were collected each from Bed linings, floor and table of the three wards. The percentage distribution of $S$. aureus in the samples is shown in Figure 2.

During the study period, $S$. aureus was also isolated from the blood samples of patients admitted to ICU $(7.5 \%)$ and NICU (6.8\%) which is shown in Table 1.
Fig 2: Percentage distribution of $S$. aureus in different environment samples of ICU, NICU and POW

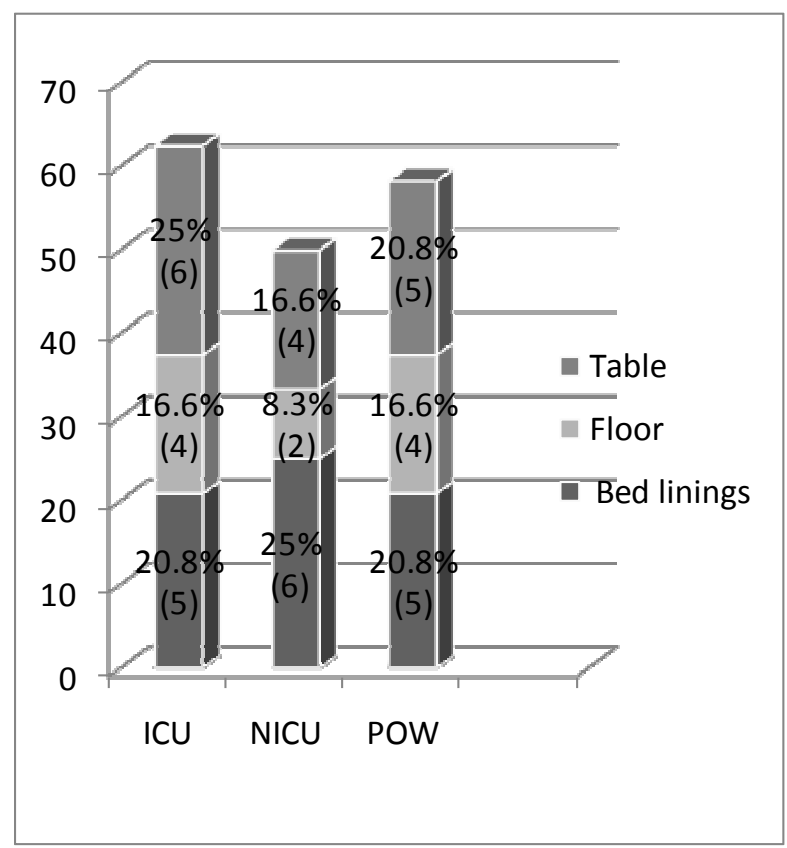

Table1: Occurrence of $S$. aureus on blood sample of patients in different wards

\begin{tabular}{|c|c|c|}
\hline Ward & $\begin{array}{c}\text { Total number } \\
\text { of Patients }\end{array}$ & $\begin{array}{c}\text { Occurrence } \\
\text { of } \text { S. aureus }\end{array}$ \\
\hline ICU & 53 & $4(7.5 \%)$ \\
\hline NICU & 29 & $2(6.8 \%)$ \\
\hline POW & 32 & - \\
\hline Total & 114 & 6 \\
\hline
\end{tabular}

Table 2: Antibiotic sensitivity pattern for $S$. aureus isolated from environmental samples

\begin{tabular}{|l|l|l|}
\hline Antibiotics & Sensitive & Resistant \\
\hline Ampicillin & $100 \%$ & $0 \%$ \\
\hline Vancomycin & $100 \%$ & $0 \%$ \\
\hline Chloramphenicol & $100 \%$ & $0 \%$ \\
\hline Gentamicin & $100 \%$ & $0 \%$ \\
\hline Oxacillin & $100 \%$ & $0 \%$ \\
\hline Meropenam & $100 \%$ & $0 \%$ \\
\hline Cotrimoxazole & $100 \%$ & $0 \%$ \\
\hline Amikacin & $100 \%$ & $0 \%$ \\
\hline
\end{tabular}


Antibiotic sensitive test for $S$. aureus isolated from environmental samples is presented in table 2 .

Nosocomial infection is a global concern. Infection acquired in health care settings are among the major causes of death and increased morbidity among hospitalized patients [2]. Five to ten percentage of hospitalized patients develop nosocomial infections [8]. A prevalence survey conducted under WHO in 55 hospitals of 14 countries representing 4 WHO regions (Europe, Eastern Mediterranean, South East Asia and Western Pacific) showed an average of $8.7 \%$ of hospitalized patients had nosocomial infection. In our study also, from a total of 216 environmental samples collected during from ICU, NICU, Post operative ward, $18.98 \%$ samples showed the presence of $S$. aureus.

S. aureus is one of the commonest organisms causing HAI. It is spread from nasal carrier, skin and inanimate objects. These organisms are present in the air, dust and surfaces where they may survive along with fungal and bacterial spores and so are the major environmental inhabitants [8]. In our study $18.9 \%$ of the samples showed the presence of $S$. aureus in them. Similarly, Banjara et al. have reported $S$. aureus to be the predominating isolate of indoor air of the hospital [9]. Another study by Weinstein has shown that $S$. aureus together with Coagulase negative Staphylococcus aureus and Enterococci accounted for $24.9 \%$ of nosocomial infections [10]. Similar study done at Tribhuvan University Teaching Hospital has shown that $S$. aureus as one of the commonest organisms prevailing in the hospital settings [11]. Over the past 50 years, $S$. aureus has acquired resistance to previously effective antimicrobials including penicillinase resistant methicillin [12]. In our study, all the strains of $S$. aureus were sensitive to the antibiotics tested and none were detected to be MRSA.

S. aureus can also cause nosocomial bacterimia [13]. In our study, S. aureus was isolated from blood of $7.5 \%$ patients in ICU and $6.8 \%$ patients in NICU. This is an indication that these strains may be the same as those prevalent in hospital environment giving rise to HAI. However, further confirmation by molecular method is essential to prove the strain identity.

The inanimate environment of the hospital comprising of contaminated air, water, food, soiled linen, hospital waste, used equipments, table, floor acts as an important source of HAI [8]. In this study also, samples were taken from bed linings, floor and table. Out of 72 samples collected from bed linings of the three wards, the occurrence of $S$. aureus was higher in NICU (25\%) than in ICU and POW (each 20.8\%). $S$. aureus was found higher in ICU and POW (each $16.6 \%)$ than in NICU (8.3\%). Among the three, working table of POW showed higher prevalence of $S$. aureus (25\%).

Studies have shown that highest prevalence of nosocomial infection occur in ICU, NICU, POW [2]. In our study also, S. aureus was detected in all the three wards; $20.8 \%, 16.6 \%, 19.4 \%$ respectively. This is not a good indication, as all the three wards admit very sensitive patient who has even higher chances of acquiring nosocomial infections. Quality check of the three units regarding sterility and aseptic technique will help to control the prevalence of $S$. aureus in the hospital environment and thereby reducing the outbreak of HAI.

\section{CONCLUSION:}

S. aureus is a common organism found in hospit- 
al environment which is also a leading cause of HAI. In our study also, $S$. aureus was found in different environmental samples of ICU, NICU, POW. Effective control of these organisms before it causes break out of HAI is essential. Therefore, regular study like this, will help to guide hospital for quality control policy. During the study period, $S$. aurues was also found in the blood samples of patients in ICU and NICU. If these strains are same as those found in hospital environment, which can be confirmed by molecular method, it will indicate a serious problem of nosocomial infection. Hence, regular correlation between quality control report of hospital environment and isolates from patients will help to identify the association of similar source of infectious organisms. This helps to manage HAI before it becomes a serious outbreak.

\section{ACKNOWLEDGEMENT}

Authors would like to thank KEC and Kist Management for their support in carrying out the research.

\section{REFERENCES}

1. Mayon White RT. An international survey of the prevalence of hospital acquired infection. J Hosp Infect 1991; 18 (supplement A): 43-8.

2. WHO/CDS/CSR/EPH. Prevention of hospital acquired infection; A practical guide $2^{\text {nd }}$ edition. WHO/CDS/CSR/EPH/2002.12.

3. MMWR, CDC. Public health focus: surveillance prevention and control of nosocomial infections. MMWR, CDC 1992; 41:1-12.

4. Lamichhane DR, Shrestha P. Prevention of nosocomial infection in TUTH. A report submitted to Nepal Health Research Council, 2001.

5. Rai SK, Pokhrel BM, Tuladhar NR, Khadka JB, Upadhyay MP. Methicillin resistant coagulase negative staphylococci. J Inst Med 1987; 9:23-8.
6. Cole A, Tahk, OrenA, Determinants of Staphylococcus aureus Nasal carriage. Clin Diag Lab Immune 8:164-9 2001

7. Kluytmans JA, Wertheim HF. Nasal carriage of Staphylococcus aureus and Prevention of nosocomial infections. Infection 2005; 33:3-8.

8. Greenwood D, Stack RCB, Peuthrer JK. Medical microbiology. A guide to microbial infections, pathogenesis and immunity, Laboratory diagnosis and control 13 $3^{\text {th }}$ edition, ELBS 1997

9. Banjara MR. Study of air, water and wound infection in different wards of teaching hospital. A dissertation submitted to the central department of microbiology, TU Kirtipur, 2002.

10. Weinstein RA. Nosocomial infection Update. Emerging Infectious diseases 1998; 4 (3): Special Issue.

11. Pokharel BM, Rawal S, Joshi HH and Kubo T. Bacteriological study at Tribhuvan University Teaching Hospital, Kathmandu, Nepal. J Inst of Med 1993; 15: 217-21.

12. Duckworth GJ. Diagnosis and management of MRSA infection. BMJ 1993; 307:1049-52.

13. Richard MJ, JR Edwards, DH Colver et al. Community acquired MRSA in pediatric ICU in the USA. Pediatrics1999; 103:39. 